\title{
AVALIAÇÃO DE DIFERENTES MATERIAIS SUPORTE PARA APLICAÇÃO NA BIOFILTRAÇÃO PARA REMOÇÃO DE ODOR DE SISTEMAS DE ESGOTOS SANITÁRIOS
}

\author{
M. C. FLORES ${ }^{1}$, G. P. SANCINETTI ${ }^{1}$ e M. V. RODRIGUES ${ }^{1}$ \\ ${ }^{1}$ Universidade Federal de Alfenas, Instituto de Ciência e Tecnologia \\ E-mail para contato: marcos.rodrigues@unifal-mg.edu.br
}

\begin{abstract}
RESUMO - Os odores produzidos durante o tratamento dos esgotos sanitários causam incômodos, poluem a atmosfera e podem ser tóxicos (ex.: gás sulfídrico). A biofiltração aeróbia dos gases pode ser uma alternativa aos processos físico-químicos comumente utilizados na indústria devido a sua eficiência, além do baixo custo de instalação e manutenção. Este trabalho avaliou a adesão microbiológica nos seguintes materiais suportes: bagaço da cana-de-açúcar, lasca de madeira, espuma de poliuretano e partículas de PET para posterior aplicação na biofiltração para remoção de odores. Os valores médios de porcentagem de retenção de biomassa nos materiais foram: bagaço da cana-de-açúcar 26,2 +/- 6,5\%; lasca de madeira 34,6 +/- 4,1\%; espuma de poliuretano $62,5+/-19,0 \%$ e partículas de PET 1,0 +/- 0,6\%. Observou-se que o a espuma de poliuretano apresentou melhores resultados de retenção de biomassa enquanto os materiais orgânicos apresentaram baixa adesão.
\end{abstract}

\section{INTRODUÇÃO}

A emissão de odor tem impacto negativo sobre a população vizinha em sistemas de esgotos sanitários e, é a principal preocupação em relação à instalação de Sistemas de Tratamento de Esgoto (ETE) em regiões urbanas e rurais. Devido ao número crescente de queixas relacionadas ao meu cheiro, as normas ambientais tem sido rigorosas com objetivo de reduzir as emissões de odor em sistemas de tratamento de esgoto (Silva, 2008, Lebrero et al., 2011).

As tecnologias que podem ser usadas para remoção de odor são os processos físicos, químicos e biológicos. Os sistemas biológicos têm sido bem aceitos devido à sua alta eficiência, baixos custos operacionais e ausência de resíduos perigosos como produtos finais. A biofiltração tem se destacado como processo de desodorização por ser uma tecnologia que não utiliza produtos químicos e não produz resíduos perigosos ao meio ambiente. Os biofiltros são fáceis de projetar e construir e, relativamente baratos. Um dos aspectos mais importantes no projeto de biofiltros é a escolha do material suporte. A seleção do material suporte adequado deve considerar vários aspectos, além daqueles relacionados com as propriedades físicas e químicas do material (Marín et al., 1999). 


\section{OBJETIVOS}

O objetivo principal deste trabalho foi avaliar a adesão microbiológica em diferentes materiais (orgânicos e inertes) para posterior aplicação na biofiltração para remoção de odores de sistemas de tratamento de esgotos sanitários. Foram avaliados os seguintes materiais suportes: bagaço da cana-de-açúcar, lasca de madeira, espuma de poliuretano e partículas de PET.

\section{REVISÃO BIBLIOGRÁFICA}

O processo de tratamento de esgotos produz odores que podem causar incômodo para a população e contribuem significativamente para a poluição atmosférica. Gases efluentes de processos industriais são quase sempre tratados por processos físico-químicos como lavadores de gases, colunas de absorção, e condensação; no entanto, o tratamento biológico destes gases tem aumentado significativamente nas últimas décadas devido a altas eficiências de remoção de gases tóxicos como pelo baixo custo de instalação e manutenção (Burgess et al 2001).

\subsection{Odor}

O odor é usualmente caracterizado com base em detecção, intensidade, qualidade e aceitabilidade (EPA, 2001). A emissão de odor afeta a qualidade de vida, levando a stress psicológico e sintomas como insônia, perda de apetite e comportamento estranho. Em sistemas de tratamento, o odor resulta em imagem ruim e reclamações por parte da população (Gostelow et al, 2001). A produção dos compostos odorantes tem origem, em compostos sulfurados ou azotados, incluindo o ácido sulfídrico, que se forma em consequência da redução do sulfato, que é a principal forma sob a qual o enxofre se apresenta nas águas residuárias e, também, da decomposição anaeróbia da matéria orgânica que potencializa a liberação de mercaptanas e de amoníaco (Silva, 2008). As condições de $\mathrm{pH}$, tipo de precursor e potencial de oxi-redução determinam a formação de determinado composto odorante. Em condição anaeróbica o ácido sulfúrico $\left(\mathrm{H}_{2} \mathrm{~S}\right)$ é um dos componentes mais comumente encontrados nas emissões gasosas proveniente da redução biológica do sulfato $\left(\mathrm{SO}_{4}{ }^{2-}\right)$ ou tiosulfato (Chernicharo, 2010).

\subsection{Processos de tratamento de odores}

Existem dois métodos pelos quais se pode controlar a emissão de gases odorantes. O método indireto em que o controle se dá por modificação do equipamento/processo, manutenção dos equipamentos e alteração de materiais, enquanto, o método direto o controle inclui técnicas destrutivas como incineração, biofiltração e técnicas reparativas, como absorção, adsorção, condensação e recuperação por membranas (Khan e Ghoshal, 2000). Segundo Frechen (1994) existem três diferentes princípios para desodorização de ar contaminado.

Processos físicos: Nos processos físicos as concentrações de contaminantes do ar não são eliminadas, porém, ocorre a transferência dos poluentes para um novo fluido a ser tratado. Seus principais processos são absorção e adsorção (Yuomono, 2004). 
Processos Químicos: A remoção do odor consiste na reação entre o composto gasoso odorante e o reagente químico adicionado. Esta forma de tratamento altera a concentração do componente odorante e consequentemente diminui a sua emissão (FNDAE, 2004).

Processos Biológicos: As tecnologias biológicas possuem simples operação, baixo custo e não agridem o meio ambiente. Os principais parâmetros que afetam o biofiltro são: material suporte, umidade do leito, $\mathrm{pH}$, temperatura, concentração dos poluentes, microrganismos e nutrientes (Tacla, 2004).

Nos processos biológicos o tratamento de compostos voláteis orgânicos e inorgânicos que possuem maus odores ocorre por meio de uma fase líquida conhecida como biofilme que transfere o gás contaminado para superfície das partículas do material suporte. Após a adesão começa o processo de degradação aeróbia realizada pelos microrganismos que convertem os poluentes em compostos simples como dióxido de carbono e água (Belli Filho et al., 2001; Tacla, 2004).

\subsection{Materiais suporte}

De acordo com Silva (2008), o material suporte deve ser responsável pela formação do biofilme sobre a sua superfície, além de reservar água e nutrientes para os microrganismos. Portanto, deve possuir a seguintes características: proporcionar ótimas condições de umidade para manter a atividade microbiana entre 40 e $60 \%$, assim, deve possuir elevada capacidade de retenção de umidade; permitir a distribuição uniforme ao longo do leito do gás poluído, para isso o material precisa apresentar porosidade maior que $80 \%$, e diâmetro maior que 4,0 $\mathrm{mm}$. Pode-se citar ainda as condições de $\mathrm{pH}$ que deve ser mantido entre 7-8 para promover a oxidação biológica; baixo custo; e vida longa (resistente às operações mecânicas e quimicamente inerte e estável).

\section{MATERIAIS E MÉTODOS}

\subsection{Materiais}

Inóculo: Foi utilizado lodo ativado proveniente de um reator aeróbio da empresa M\&G Fibras Brasil S.A para aumentar a umidade dos materiais suporte e enriquecê-los com microrganismos.

Solução nutriente: De acordo com Lebrero et al. (2001), para garantir que os nutrientes básicos fossem fornecidos ao desenvolvimento dos microrganismos foi elaborada um litro de solução que consistia em $(\mathrm{g} / \mathrm{L})$ de: $\mathrm{Na}_{2} \mathrm{HPO}_{4} \cdot 12 \mathrm{H}_{2} \mathrm{O}, 6,15 ; \mathrm{KH}_{2} \mathrm{PO}_{4}, 1,52 ; \mathrm{NH}_{4} \mathrm{Cl}, 0,81$; $\mathrm{MgCl}_{2} \cdot 6 \mathrm{H}_{2} \mathrm{O}, 0,17 ; \mathrm{CaCl}_{2}, 0,038 ;$ e $10(\mathrm{ml} / \mathrm{L})$ de traços de solução contendo (g/L): EDTA, 0,$5 ; \mathrm{FeSO}_{4} \cdot 7 \mathrm{H}_{2} \mathrm{O}, 0,2 ; \mathrm{ZnSO}_{4} \cdot 7 \mathrm{H}_{2} \mathrm{O}, 0,01 ; \mathrm{MnCl}_{2} \cdot 4 \mathrm{H}_{2} \mathrm{O}, 0,003 ; \mathrm{H}_{3} \mathrm{BO}_{3}, 0,03 ; \mathrm{CoCl}_{2} \cdot 6 \mathrm{H}_{2} \mathrm{O}$, 0,$02 ; \mathrm{CuCl}_{2} \cdot 2 \mathrm{H}_{2} \mathrm{O}, 0,001 ; \mathrm{NiCl}_{2} \cdot 6 \mathrm{H}_{2} \mathrm{O}, 0,002 ; \mathrm{NaMoO}_{4} \cdot 2 \mathrm{H}_{2} \mathrm{O}, 0,003$.

Material suporte: Os materiais suporte empregados foram o bagaço de cana-de-açucar, cascas de madeira coletadas em uma madeireira da região, grânulos de PET, fornecidos pela M\&G Fibras Brasil S.A., e espuma de poliuretano. 


\subsection{Procedimento experimental}

Os materiais passaram por um processo de preparação demostrado na Figura 1.

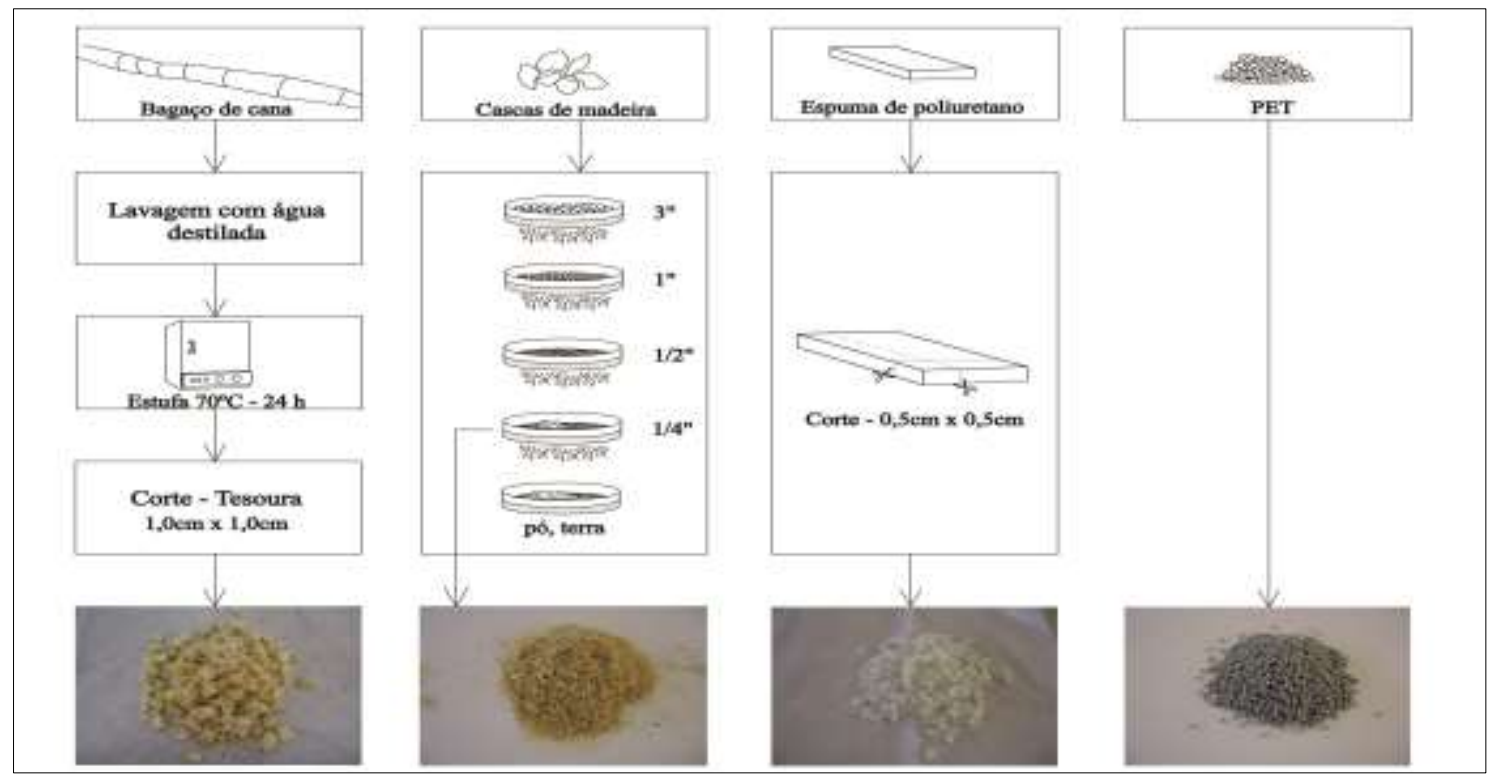

Figura 1 - Preparação do material suporte.

Utilizou-se um béquer, no qual o material suporte, a solução nutriente e o lodo ativado foram inseridos. A agitação era realizada com o auxílio de um agitador magnético. A montagem é mostrada na Figura 2.

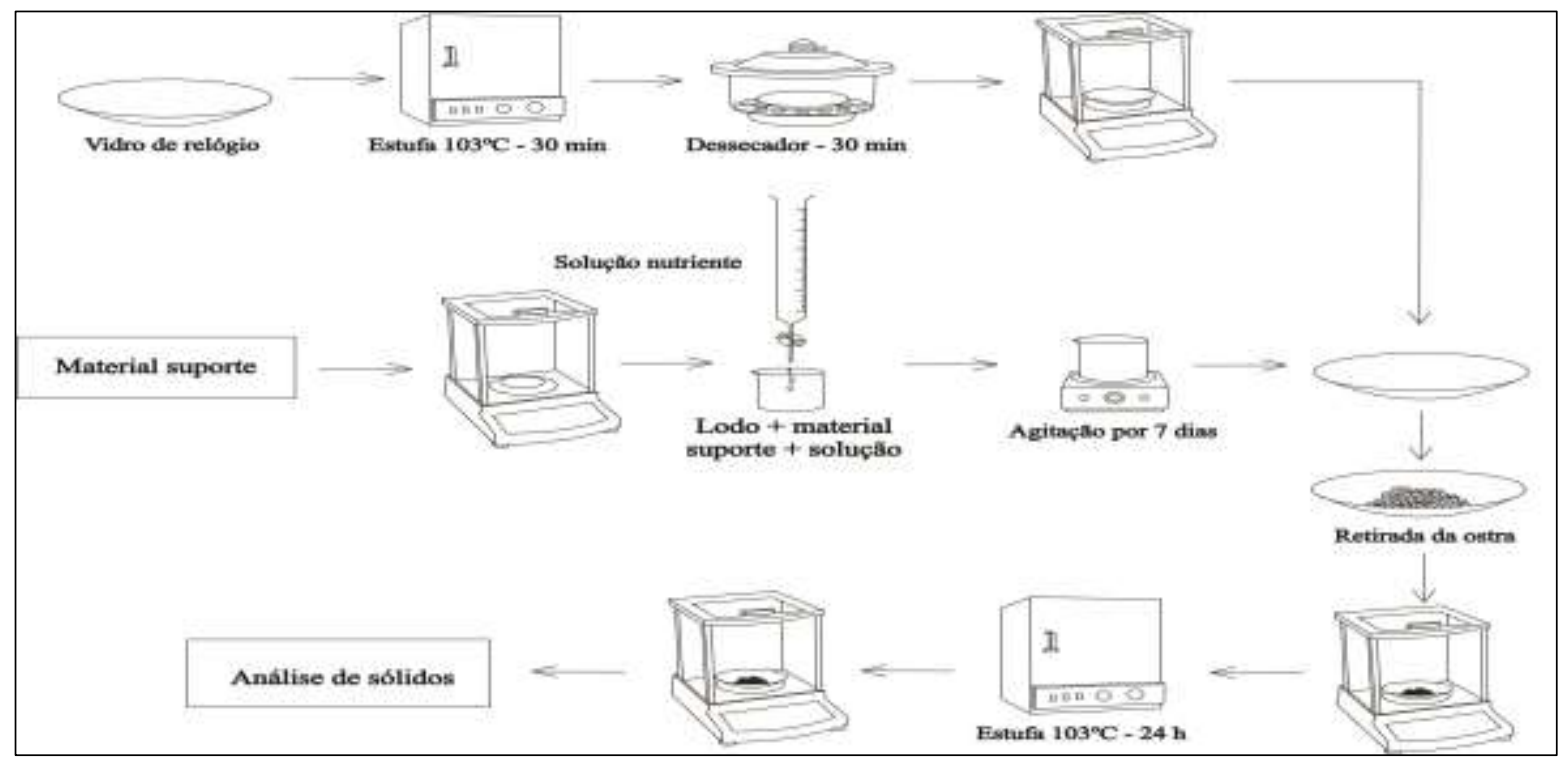

Figura 2 - Elaboração do experimento.

Os ensaios foram feitos em triplicata para cada material suporte de acordo com as condições experimentais 1 e 2 . Para os materiais orgânicos, foram feitos testes em branco para avaliar a interferência do suporte nas análises de sólidos totais. 
$\checkmark$ Condição 1: $20 \%$ material suporte, $75 \%$ de lodo ativado e $5 \%$ de nutriente;

$\checkmark$ Condição 2 - 50\% de material suporte, $45 \%$ de lodo ativado e $5 \%$ de nutriente;

As porcentagens descritas em massa. Foram utilizados béqueres de $500 \mathrm{ml}$ sendo $50 \%$ do seu volume foi preenchido.

\subsection{Análises}

As análises realizadas foram:

Sólidos totais: o teste foi feito em estufa com temperatura controlada em $103^{\circ} \mathrm{C}$. A amostra foi colocada por período de 24 horas até peso constante. Obteve-se a porcentagem de umidade da amostra analisada, através da diferença do peso da amostra no início e após 24 horas. A porcentagem de sólidos totais é dada pela diferença de $100 \%$ e a umidade.

Porosidade: Determinada em bureta com a relação entre os volumes de suporte e volume de água adicionada;

Adsorção: a análise foi feita a partir dos dados de sólidos totais retirando-se o peso seco do suporte.

\section{RESULTADOS E DISCUSSÃO}

A biofiltração da corrente de gás contaminada só é possível devido à presença dos microrganismos impregnados no suporte. Os resultados obtidos para os diferentes materiais avaliados estão apresentados na Tabela 1.

Tabela 1 - Resultados de sólidos totais, porosidade e adsorção para os materiais suporte avaliados.

\begin{tabular}{|c|c|c|c|}
\hline Materiais & Parâmetros & Condição 1 & Condição 2 \\
\hline \multirow{2}{*}{$\begin{array}{c}\text { Bagaço de } \\
\text { cana }\end{array}$} & \%Porosidade & $78,2 \pm 1,8$ & $77,7 \pm 1,7$ \\
\cline { 2 - 4 } & \%Adsorção & $21,4 \pm 4,8$ & $31,0 \pm 3,9$ \\
\hline \multirow{2}{*}{$\begin{array}{c}\text { Cascas de } \\
\text { madeira }\end{array}$} & \%Porosidade & $89,4 \pm 0,5$ & $88,2 \pm 0,7$ \\
\cline { 2 - 4 } & \%Adsorção & $33,4 \pm 6,1$ & $35,8 \pm 0,9$ \\
\hline $\begin{array}{c}\text { Espuma de } \\
\text { poliuretano }\end{array}$ & \%Porosidade & $73,0 \pm 1,2$ & $75 \pm 1,4$ \\
\cline { 2 - 4 } & \%Adsorção & $62,4 \pm 3,1$ & $74,1 \pm 12,7$ \\
\hline \multirow{2}{*}{ PET } & \%orosidade & $33,8 \pm 0,2$ & $33,1 \pm 0,2$ \\
\cline { 2 - 4 } & \%Adsorção & $0,7 \pm 0,2$ & $1,3 \pm 0,8$ \\
\hline
\end{tabular}

Os valores encontrados indicam que apenas o PET não apresentou parâmetros esperados para um material ser utilizado como meio suporte. Características recomendadas para 
materiais suporte de biofiltros devem ter $50 \%$ de teor de sólidos e porosidade superior a $60 \%$ (Silva, 2008).

A quantidade de lodo aderido em cada material para as duas condições experimentais está mostrada na Figura 3.

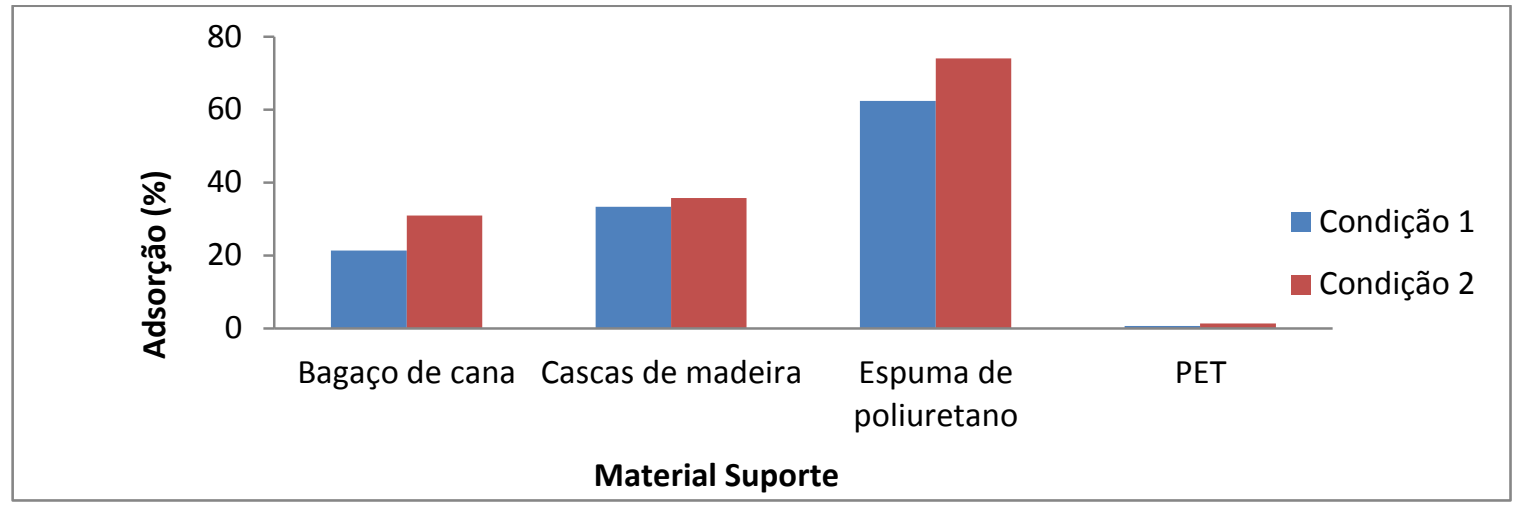

Figura 3 - Adesão dos microrganismos ao material suporte.

Sabe-se que o lodo ativado é rico em microrganismos, assim, é o responsável pela inoculação do material suporte. A espuma de poliuretano destacou-se entre as demais com elevada capacidade de adesão dos microrganismos à sua superfície independente das condições experimentais adotadas.

A condição 2 possuía menor quantidade de inoculo para uma maior quantidade de material suporte. Nesta condição a aderência dos microrganismos foi mais significativa possivelmente devido a maior superfície disponível. A menor adesão do PET era esperada devido a superfície lisa deste material.

A espuma de poliuretano foi o material que apresentou melhores resultados de adesão. Mas é importante destacar que o bagaço de cana e a casca de madeira mostraram viabilidade para sua aplicação.

\section{CONCLUSÃO}

Ao analisarmos apenas a adesão dos microrganismos à superfície dos materiais suporte, a espuma de poliuretano apresentou os melhores resultados, enquanto o bagaço de cana e a casca de madeira mostraram ser viáveis para utilização como suporte em biofiltro.

Para continuidade do trabalho será avaliada a passagem do gás contaminado por um biofiltro para realizar a análise da interferência na quantidade de microrganismo aderido na remoção de odor.

\section{REFERÊNCIAS}

BELlI FILHO, P.; COSTA, R. H. R.; GONÇALVES, R. F.; CORAUCCI FILHO, B.; LISBOA, H. M. Tratamento de Odores em Sistemas de Esgotos Sanitários. In:Pós-tratamento de efluentes de reatores anaeróbios - Belo Horizonte: PROSAB,2001. Cap. 8, p.455-490. 
BURGESS, J.E.; SIMON, A.P.; STUET, R.M. Developments in odour control and waste gas treatment biotechnology: A review. Biotech. Ad., v.19, p,35-63, 2001.

CHERNICHARO, C.A.L. Alternativas para o controle de emissões odorantes em reatores anaeróbios tratando esgoto doméstico. Eng. Sanit. Ambient, v.15, n.3, p.229-236, 2010.

FNDAE - Fonds National Pour Le Developpement Des Adductions D'eau. Lutte Contre Les Odeurs De L'assainissement. Rédigé par Celine Debrieu. Of. Int. de I'Eau SNIDE. Doc. Tech. $\mathrm{n}^{\mathrm{o}} 13,02 / 07 / 2004$.

FRECHEN, F. B. Odour emissions of wastewater treatment plants in West Germany.W. Sc. Tech., v. 30, p.35-46, 1994.

GOSTELOW, P.; PARSONS, S.A.; STUETZ, R.M. Odour Measurements for Sewage Treatment Works. Wat. Res. vol. 35, nº 3, p. 579-597, 2001.

KHAN, F. I.; GHOSHAL, A. K. Removal of volatile organic compounds from polluted air. J. loss prev. in the proc. Ind., v. 13: p. 527-545, set. 2000.

LEBRERO, R.; RODRIGUEZ, E.; GARCIA-ENCINA, P.A.; MUÑOZ, R. A comparative assessment of biofiltration and activated sludge diffusion for odour abatement. J. Haz. Mat., p. 622-630, 2011.

MARÍN, P.; ALKALAY, D.; GUERRERO, L.; CHAMY, R.; SCHIAPPACASSE, M.C. Design and startup of an anaerobic fluidized bed reactor. Wat. Sc. Tech.y, v. 40, n. 8, p. 6370, 1999.

McNEVIN, D.; BARFORD, J. Biofiltration as an odour abatement strategy. Bioc. Eng. J., v.5, p.231-242, 2000.

SILVA, M.B. Influência do tipo de meio suporte no desempenho de biofiltros aplicados à remoção de $\mathrm{H}_{2} \mathrm{~S}$ do ar atmosférico em sistemas de esgoto sanitário. Vitória, UFES, (Dissertação de Mestrado) 2008.

TACLA, R.M.B; Aproveitamento de resíduos industriais para a biofiltração do sulfeto de hidrogênio, Curitiba, UFPR, (Dissertação de Mestrado), 162p, 2004.

YUMONO, A. S.; LAMMERS, P. S. Odor Pollution in the Environment and the Detection Instrumentation. Agricultural Engineering International: the CIGR J. Sc. Res. Dev., vol 4, 2004. 